\title{
AN ESTIMATE OF SECTIONAL CURVATURES OF HYPERSURFACES WITH POSITIVE RICCI CURVATURES
}

\author{
by JU SEON KIM and SANG OG KIM
}

(Received 21st September 1993)

\begin{abstract}
Let $M$ be a hypersurface in Euclidean space and let the Ricci curvature of $M$ be bounded below by some nonnegative constant. In this paper, we estimate the sectional curvature of $M$ in terms of the lower bound of Ricci curvature and the upper bound of mean curvature.
\end{abstract}

1991 Mathematics subject classification: 53A07, 53C20

\section{Introduction}

It is well-known that if Ricci curvature of a complete Riemannian manifold $M$ is bounded below by a positive constant then it is compact. If $M$ is a hypersurface then the principal curvatures are defined at each point. In this paper, we estimate the bounds of sectional curvature in terms of the lower bound of Ricci curvature and the upper bound of mean curvature. Throughout this paper, $M^{n}$ or $M$ is an $n$-dimensional complete connected hypersurface embedded in $R^{n+1}$, where $n \geqq 3$. And we denote the mean curvature (the trace of the second fundamental form) by $H$ and the sectional curvature by $K$.

\section{Estimates of sectional curvature}

Lemma 2.1. The Ricci curvature is nonnegative on $M^{n}$ if and only if the sectional curvature is nonnegative on $M^{n}$.

Proof. Fix $p \in M$. Let $\left\{e_{i}\right\}_{1 \leqq i \leqq n},\left\{\lambda_{i}\right\}_{1 \leqq i \leqq n}$ be the principal vectors and the corresponding principal curvatures respectively, at $p$. We may assume that $\lambda_{1} \leqq \cdots \leqq \lambda_{n}$. Let $H$ be the mean curvature at $P$, that is, $H=\lambda_{1}+\cdots+\lambda_{n}$. We may assume that $H \geqq 0$. Suppose $\operatorname{Ric}(x, x)=0$ for each unit vector $x \in T_{p}(M)$. Then $\operatorname{Ric}\left(e_{i}, e_{i}\right)=\lambda_{i}\left(H-\lambda_{i}\right)=0$. Hence either all $\lambda_{i}$ 's are 0 or only one $\lambda_{i}$ is nonzero. Hence the sectional curvature is 0 . Suppose $\operatorname{Ric}(x, x)>0$ for each unit vector. We may assume that $H \geqq 0$. If $\lambda_{i}<0$ for some $i$, then $\operatorname{Ric}\left(e_{i}, e_{i}\right)=\lambda_{i}\left(H-\lambda_{i}\right)<0$, which is a contradiction. So it follows that all $\lambda_{i}^{\prime} s$ are positive. Let $x, y$ be unit vectors with $x \perp y$ and $x=\sum_{i=1}^{n} a_{i} e_{i}, y=\sum_{j=1}^{n} b_{j} e_{j}$. Then

$$
K(x, y)=\langle A(x, x), A(y, y)\rangle-\langle A(x, y), A(x, y)\rangle
$$




$$
\begin{aligned}
& =\left(\sum_{i=1}^{n} a_{i}^{2} \lambda_{i}\right)\left(\sum_{j=1}^{n} b_{j}^{2} \lambda_{j}\right)-\left(\sum_{i=1}^{n} a_{i} b_{i} \lambda_{i}\right)^{2} \\
& =\sum_{i<j}\left(a_{i} b_{j}-a_{j} b_{i}\right)^{2} \lambda_{i} \lambda_{j},
\end{aligned}
$$

where $A$ is the second fundamental form. Hence the sectional curvature $K$ at $p$ is positive. The proof of the other direction is clear.

Corollary 2.2. If $a \leqq R i c(x, x)$ for some positive constant $a$ and for each unit vector $x \in T M$, then $M$ is diffeomorphic to $S^{n}$.

Proof. Since $0<a \leqq \operatorname{Ric}(x, x), M$ is compact. And the second fundamental form $A$ is definite everywhere on $M$ by Lemma 2.1. So the spherical map of Gauss $M \rightarrow S^{n}$ is a diffeomorphism ([3]).

The following lemma is a corollary of the Gauss equation, but we give another elementary proof.

Lemma 2.3. If $a \leqq R i c(x, x)$ for some positive constant $a$ and for each unit vector $x \in T M$, then $\left(n^{2} a /(n-1) \leqq H^{2}\right.$, where $H$ is the mean curvature of $M$.

Proof. Let $\left\{\lambda_{i}\right\},\left\{\boldsymbol{e}_{i}\right\}$ denote as in the proof of (2.1) above. We may assume that $H>0$. Then all $\lambda_{i}^{\prime} s$ are positive. Since $a \leqq \lambda_{1}\left(H-\lambda_{1}\right)$ and $H>0$, we have

$$
\lambda_{1} \geqq \frac{H-\sqrt{H^{2}-4 a}}{2}
$$

and $H^{2} \geqq 4 a$. Since $\lambda_{1} \leqq H / n$, we have

$$
\frac{n\left(H-\sqrt{H^{2}-4 a}\right)}{2} \leqq n \lambda_{1} \leqq H,
$$

that is,

$$
n\left(H-\sqrt{H^{2}-4 a}\right) \leqq 2 H
$$

So we have

$$
H^{2} \geqq \frac{n^{2} a}{n-1}
$$

Theorem 2.4. If $o<a \leqq R i c(x, x)$ for all unit vectors $x \in T M$ and the mean curvature $|H| \leqq H_{0}$ for some constant $H_{0}$, then we have 


$$
0<\frac{H_{0}\left(H_{0}-\sqrt{H_{0}^{2}-4 a}\right)}{2}-a \leqq K<\frac{H_{0}^{2}}{4},
$$

where $K$ is the sectional curvature of $M^{n}$.

Proof. Let $\left\{\lambda_{i}\right\},\left\{e_{i}\right\}$ be as in the proof of (2.1) above. Let $x, y$ be unit vectors with $x \perp y$ and $x=\sum_{i=1}^{n} a_{i} e_{i}, y=\sum_{j=1}^{n} b_{j} e_{j}$. Then by the proof of (2.1), we have

$$
K(x, y)=\sum_{i<j}\left(a_{i} b_{j}-a_{j} b_{i}\right)^{2} \lambda_{i} \lambda_{j}
$$

Since $\sum_{i=1}^{n} a_{i} e_{i}, \sum_{j=1}^{n} b_{j} e_{j}$ are orthonormal vectors,

$$
\begin{aligned}
1 & =\left(a_{1}^{2}+\cdots+a_{n}^{2}\right)\left(b_{1}^{2}+\cdots+b_{n}^{2}\right)-\left(a_{1} b_{1}+\cdots+a_{n} b_{n}\right)^{2} \\
& =\sum_{i \neq j} a_{i}^{2} b_{j}^{2}-2 \sum_{i<j} a_{i} b_{i} a_{j} b_{j} \\
& =\sum_{i<j}\left(a_{i} b_{j}\right)^{2}-2 \sum_{i<j} a_{i} b_{j} a_{j} b_{i}+\sum_{i<j}\left(a_{j} b_{i}\right)^{2} \\
& =\sum_{i<j}\left(a_{i} b_{j}-a_{j} b_{i}\right)^{2} .
\end{aligned}
$$

We may assume that $H>0$. Then $0<\lambda_{1} \leqq \lambda_{2} \leqq \cdots \leqq \lambda_{n}$ by (2.1). Then we have $\lambda_{1} \lambda_{2} \leqq$ $K(x, y) \leqq \lambda_{n-1} \lambda_{n}$. By the proof of $(2.3)$,

$$
\frac{\left.H-\sqrt{H^{2}-4 a}\right)}{2} \leqq \lambda_{1},
$$

so that

$$
\frac{H\left(H-\sqrt{H^{2}-4 a}\right)}{2}-a \leqq \lambda_{1}^{2},
$$

and

$$
H^{2} \geqq \frac{n^{2} a}{n-1}
$$

If we define

$$
g(X)=\frac{X-\sqrt{X^{2}-4 a X}}{2}
$$


then $g^{\prime}(X)<0$ when $X>4 a$. By letting $H^{2}=X$, we see that

$$
\frac{H\left(H-\sqrt{H^{2}-4 a}\right)}{2}=\frac{H^{2}-\sqrt{H^{4}-4 a H^{2}}}{2}
$$

is monotone decreasing from $n a /(n-1)$ to $a$ on the interval $\left[\sqrt{n^{2} a /(n-1)}, \infty\right)$ as a function of $H$. So we have

$$
0<\frac{H_{0}\left(H_{0}-\sqrt{H_{0}^{2}-4 a}\right)}{2}-a \leqq \lambda_{1}^{2} \leqq \lambda_{1} \lambda_{2} \leqq K .
$$

On the other hand,

$$
\lambda_{n-1} \lambda_{n}<\left(H-\lambda_{n}\right) \lambda_{n} \leqq \frac{\left(H-\lambda_{n}+\lambda_{n}\right)^{2}}{4}=\frac{H^{2}}{4} \leqq \frac{H_{0}^{2}}{4},
$$

so we have $K(x, y)<H_{0}^{2} / 4$.

\section{REFERENCES}

1. J. Cheeger and D. G. Ebin, Comparison theorems in Riemannian geometry (1975).

2. H. JACOBwITZ, Isometric embedding of a compact Riemannian manifold into Euclidean space, Proc. Amer. Math. Soc. 40 (1973), 245-246.

3. S. Kobayashi and K. Nomizu, Foundations of Differential Geometry (1969).

Department of Mathematics

Seoul National University 151-742

KOREA
Department of Mathematics HaLLYM UnIVERSITY, 200-702 KOREA 